\title{
Références bibliographiques du dossier : » école primaire, école de base »
}

Prunelle Charvet

\section{OpenEdition}

1 Journals

Édition électronique

URL : http://journals.openedition.org/ries/1166

DOI : 10.4000/ries. 1166

ISSN : 2261-4265

Éditeur

Centre international d'études pédagogiques

\section{Édition imprimée}

Date de publication : 1 avril 2006

Pagination : 97-109

ISSN : 1254-4590

\section{Référence électronique}

Prunelle Charvet, «Références bibliographiques du dossier : » école primaire, école de base » », Revue internationale d'éducation de Sèvres [En ligne], 41 | avril 2006, mis en ligne le 16 novembre 2011, consulté le 14 novembre 2019. URL : http://journals.openedition.org/ries/1166 ; DOI : 10.4000/ries. 1166

(c) Tous droits réservés 


\title{
Références bibliographiques
}

\section{Prunelle Charvet}

\begin{abstract}
Depuis le Forum mondial sur l'éducation, qui s'est tenu à Dakar en avril 2000, l'accès de tous à un enseignement primaire de qualité est devenu une priorité mondiale. Cette bibliographie recense des études transnationales sur les politiques de développement pour l'éducation primaire et l'éducation de base, l'évaluation, les programmes, cycles et rythmes scolaires ainsi que sur la formation des maîtres. Elle propose ensuite un accès géographique à des études de cas puis quelques ouvrages de référence sur l'école primaire française.

Les publications concernant les pays impliqués dans les programmes de scolarisation primaire universelle sont le plus souvent postérieures à l'année 2000. Les autres, en particulier celles qui traitent de la situation en Europe, sont parfois moins actuelles mais restent les plus récentes sur le sujet. Des résumés des documents figurent dès lors qu'ils sont en ligne ou consultables au centre de ressources du Centre international d'études pédagogiques. Cette bibliographie a été arrêtée le 10 février 2006.
\end{abstract}

\section{Études transnationales}

\section{Politiques de développement}

Banque mondiale, Education pour tous - Initiative de mise en ouvre accélérée: accélérer les progrès vers une éducation primaire universelle de qualité : document cadre, 2004, 18 pages téléchargées du site www.worldbank.org

Ce document décrit de manière synthétique l'initiative de mise en œuvre accélérée de l'Éducation pour tous créée en 2002 pour soutenir les objectifs globaux de l'EPT et notamment accélérer les progrès nécessaires à l'achèvement de la scolarisation primaire universelle d'ici à 2015.

CARRON Gabriel, CHAU Ta Ngoc, IIPE: Institut international de planification de l'éducation/Paris, La qualité de l'école primaire dans des contextes de développement différents, UNESCO/Paris, 1998, 342 p.

Ce rapport, centré sur le fonctionnement des écoles primaires, est fondé sur une analyse comparative d'études de cas réalisées par des équipes nationales dans quatre pays: Chine, Guinée, Inde et Mexique. Après une présentation générale du projet (approche méthodologique, objectifs de la recherche), il compare les principaux résultats de cette recherche pour en dégager des réflexions sur les différents modes de fonctionnement des écoles dans des contextes locaux variables. Enfin, il résume les principales conclusions et examine leurs implications pour la planification et la gestion.

DUCRET Jean-Jacques coord., BENNET John, NEUMAN Michelle J. et al. Scolariser la petite enfance, Perspectives (Unesco), décembre 2004, vol. XXXIV, $n^{\circ} 4$, 140 pages téléchargées du site www.ibe.unesco.org

Ce dossier présente les principales contributions d'un colloque sur la scolarisation de la petite enfance, qui a réuni en 2003, à Genève, des chercheurs, des professionnels et des 
administrateurs américains, asiatiques, européens et nord-africains. Les points suivants sont particulièrement étudiés: l'âge de préscolarisation, les efforts de certains pays de l'OCDE pour mettre en place des services éducatifs de qualité, les particularités de l'Italie et de la France qui sont parmi les premiers pays à avoir développé des «écoles de la petite enfance», les politiques de préscolarisation dans des pays dotés de moins de moyens tels que l'Algérie, le Brésil, la Chine et le Maroc.

MINGAT Alain, BRUNS Barbara, RAKOTOMALALA Ramahatra, Achieving universal primary education by 2015: a chance for every child, World Bank/ Washington/États-Unis, août 2003, 241 pages téléchargées du site www-wds.worldbank.org

Afin d'évaluer les probabilités de parvenir à une éducation primaire pour tous d'ici à 2015, date fixée pour les objectifs de développement pour le millénaire, l'ouvrage examine les progrès réalisés jusqu'à présent par les pays et évalue les réformes politiques et les promesses de financements nécessaires à la poursuite de ces progrès. Des tableaux statistiques sur les indicateurs en matière d'éducation et les ressources financières figurent à la fin du document.

OCDE: Organisation de coopération et de développement économiques, Petite enfance, grands défis: éducation et structures d'accueil, OCDE/Paris, 2001, 247 p. (Enseignement et compétences)

Douze pays de l'OCDE, essentiellement européens, ont accepté de participer à l'examen thématique de la politique d'éducation et d'accueil des jeunes enfants (n'ayant pas encore atteint l'âge de l'obligation scolaire) lancé par le Comité de l'éducation de l'OCDE en 1998. Des données d'ensemble ont été recueillies, ce qui a permis l'analyse comparative tout en dégageant un certain nombre de stratégies d'action.

TOMASEVSKI Katarina, Free and compulsory education for all children: the gap between promise and performance, Novum Grafiska AB/Gothenburg/Suède, [2001]., 80 pages téléchargées du site www.right-to-education.org (Right To Education Primers)

Après avoir rappelé les obligations fondamentales des Droits de l'homme et notamment le droit à l'éducation primaire, le document présente, de manière synthétique, les engagements des gouvernements par rapport aux Droits de l'homme internationaux qui garantissent une éducation gratuite et obligatoire pour tous les enfants. La dernière partie du document liste cinquante-huit pays dans lesquels l'éducation primaire obligatoire et gratuite n'est pas complètement assurée.

Toward universal primary education: investments, incentives and institutions, United nations development program/New-York/États-Unis, 2005, 183 pages téléchargées du site www.unmillenniumproject.org

Ce rapport aborde les défis de l'éducation dans les pays en voie de développement et propose des stratégies pour accélérer le progrès et atteindre les objectifs de développement fixés par la Déclaration du millénaire. 


\title{
Évaluation de l'éducation primaire
}

\begin{abstract}
BORLAND Melvin V., HOWSEN Roy M., An Examination of the effect of elementary school size on student academic achievement, International review of education, septembre 2003, vol. 49, $n^{\circ}$ 5, pp. 463-474

À la suite des conclusions contradictoires d'études réalisées sur la relation entre la taille de l'établissement et les résultats scolaires, cet article complète l'analyse en y intégrant d'autres variables, notamment le facteur des aptitudes individuelles. Il conclut à une relation non linéaire entre les effectifs et les résultats scolaires et de ce fait, à l'existence d'un effectif optimal pour la maximalisation du rendement scolaire.
\end{abstract}

GAUTHIER Pierre-Louis coord., L'évaluation des systèmes éducatifs, Revue internationale d'éducation de Sèvres, $n^{\circ} 26$, juin 2000, 155 p.

Les différentes politiques d'évaluation et les programmes d'amélioration de l'enseignement primaire qui en découlent sont étudiées à travers l'examen des systèmes éducatifs européens (Pays-Bas, France, Allemagne, Espagne, Grande-Bretagne, Italie) et africains (Guinée, Afrique du sud).

UNESCO: Organisation des Nations-Unies pour l'éducation la science et la culture, Les enfants non scolarisés: mesurer l'exclusion de l'éducation primaire, UNESCO/Paris, 2005, 115 pages téléchargées du site www.uis.unesco.org

Le rapport s'appuie sur des données d'enquêtes menées auprès des ménages de quatre-vingts pays pour explorer les caractéristiques des enfants non scolarisés. Il présente également des preuves convaincantes des disparités dues à la richesse du ménage, au milieu de résidence et au genre.

\section{Programmes, cadres, cycles, rythmes scolaires}

ALEXANDER Robin, MARCHIVE Alain, BOROWSKI Patricket al., Aspects de l'école élémentaire, Revue française de pédagogie, 2003, $n^{\circ} 142$, pp. 5-53.

Après avoir présenté une étude comparative de l'enseignement élémentaire en Angleterre, en France, en Inde, en Russie et aux États-Unis, le dossier présente une vision ethnographique des règles de la vie scolaire.

LE MÉTAIS Joanna, International Trends in Primary Education: INCA Thematic Study No. 9, Qualifications and Curriculum Authority/Londres, juin 2003, 128 pages téléchargées du site www.inca.org.uk

L'auteur rend compte des tendances internationales en matière d'éducation primaire à partir d'études menées ces vingt dernières années sur l'éducation primaire (structure, organisation, curricula, évaluation, pratiques pédagogiques, réformes) dans dix-huit pays : Australie, Canada, Angleterre, France, Allemagne, Hongrie, Irlande, Italie, Japon, Corée, Pays-Bas, Nouvelle-Zélande, Singapour, Espagne, Suède, Suisse, États-Unis et Pays de Galles.

MOYLES Janet éd., HARGREAVES Linda éd., The primary curriculum : learning from international perspectives, Routledge \& Kegan/Londres, 1998, 240 p., tabl., graph., ill.

Ces différents contributeurs analysent les relations et tensions en éducation entre les désirs des enfants et les demandes de la société auxquels les instituteurs doivent faire face 
quotidiennement. L'interaction entre enfance, programmes d'enseignement et pratiques de classe est ici placée dans une perspective internationale. Dans la première partie, est présenté un cadre de réflexion sur les programmes du primaire, dans la seconde partie des études sur les programmes d'Afrique du Sud, Australie, Nouvelle-Zélande, Asie du Sud-Est, Europe et ÉtatsUnis.

PERRAUDEAU Michel, Les cycles et la différenciation pédagogique, A. Colin/ Paris, 1997, 169 p., bibliogr. (Formation des enseignants. Enseigner).

Les aspects théoriques de la différenciation de l'apprentissage (grands courants éducatifs, théories de Piaget et Wallon) sont d'abord abordés. Viennent ensuite un tableau des diverses méthodes de la pédagogie différenciée et une définition du concept de différenciation éclairé par les apports de P. Meirieu, A. de la Garanderie, H. Trocmé-Fabre, B.-M. Barth. Des précisions sont apportées sur le concept et la mise en œuvre des cycles.

PERRENOUD Philippe, Les cycles d'apprentissage. Une autre organisation du travail pour combattre l'échec scolaire, Presses de l'Université du Québec/SainteFoy/Canada, 2002, 204 p. (Éducation intervention).

L'auteur propose une réflexion approfondie sur le concept même de cycle d'apprentissage, sur sa mise en place et la gestion de nouveaux espaces-temps, sur l'individualisation des parcours, sur le rôle des enseignants et leur engagement dans ces nouvelles pratiques.

\section{Formation des maîtres, pratiques pédagogiques}

BERNARD Jean-Marc, Les enseignants du primaire dans cinq pays du programme d'analyse des systèmes éducatifs de la CONFEMEN: le rôle du maître dans le processus d'acquisition des élèves, IREDU/Dijon, 1999, iii-176p.

Ce rapport tente un état des lieux des données disponibles sur les maîtres du primaire dans cinq pays de la CONFEMEN (Burkina-Faso, Côte-d’Ivoire, Cameroun, Madagascar et Sénégal) afin de mieux appréhender leur rôle dans le processus d'acquisition des élèves. Deux éléments ressortent de cette analyse: le niveau de recrutement optimal des maîtres se situe au niveau du second cycle du secondaire et l'efficacité de la formation professionnelle est remise en cause.

GASPARINI Rachel, Ordres et désordres scolaires: la discipline à l'école primaire, Grasset/Paris, 2000, 285 p. (Partage du savoir).

À travers des études de cas prises dans trois classes pratiquant des pédagogies novatrices - classe Montessori, classe Freinet, classe plurielle - l'auteur propose ses réflexions à propos de la discipline scolaire et son rapport avec la relation pédagogique avec, en arrière plan, cette interrogation: «comment arriver à contraindre l'élève tout en prétendant l'éduquer à la liberté?».

GAUTHIER Pierre-Louis coord., La formation des maîtres, I. des approches contrastées, Revue internationale d'éducation de Sèvres, $n^{\circ} 20,1998,155$ p.

GAUTHIER Pierre-Louis (Dir.), La formation des maîtres, II. Des problématiques convergentes, Revue internationale d'éducation de Sèvres, $n^{\circ} 21,1999,155$ p.

Dans les deux numéros cités ci-dessus de la Revue internationale d'éducation de Sèvres, la formation des enseignants du primaire est analysée de manière critique sur quatre continents par des experts européens (Pays-Bas, Espagne, France, Allemagne, Grande-Bretagne, Italie, Pologne), asiatiques (Japon, Chine), africains (Afrique du sud, Cameroun, Guinée) et américains (États-Unis). 
GOHIER Christiane, ANADON Marta, BOUCHARD Yvon et al., La construction de l'identité professionnelle de l'enseignant dans le curriculum en formation des maîtres: l'évaluation examinée, Dossiers des sciences de l'éducation (Les), 2001, $n^{\circ}$ 6, pp. 93-104.

GONDRAND Hélène, Des archétypes de professionnalisation des professeurs d'école, 2004, Sciences de l'éducation pour l'ère nouvelle (Les), vol. 37, n² 2, pp. 105-129.

Haut conseil de l'évaluation de l'école, L'évaluation des pratiques éducatives dans les premier et second degrés, HCEE, Paris, 2002, 91 pages téléchargées du site www.ladocumentationfrancaise.fr

Après l'évaluation institutionnelle des pratiques éducatives, ce rapport présente les recherches empiriques visant à estimer leurs effets. Ces recherches fondées sur des travaux français et internationaux portent sur les pratiques éducatives au niveau des établissements et des classes.

\section{Données statistiques}

SEN Anindita, PARTELOW Lisette A., MILLER David C. et al., U. S. Department of Education, Institute of Education Sciences, National Center for Education Statistics/ Washington DC, Comparative indicators of education in the United States and other G-8 countries: 2004, National center for education statistics/Washington/ États-Unis, février 2005, 86 pages téléchargées du site http://nces.ed.gov

Ce rapport permet de décrire dans une perspective comparatiste les systèmes éducatifs des pays du G8. Les données proviennent essentiellement de trois sources: les indicateurs de l'OCDE, l'évaluation menée par l'International Association for the Evaluation of Education Achievement, l'IAE, et le Programme international pour le suivi des acquis des élèves, PISA (2000). Les 31 indicateurs retenus sont organisés en cinq parties : contexte démographique et socioéconomique de l'éducation, enseignements préélémentaire et élémentaire, enseignement secondaire, enseignement supérieur, relations entre enseignement et monde du travail.

UNESCO: Institut de statistique, Recueil de données mondiales sur l'éducation 2005 : statistiques comparées sur l'éducation dans le monde, Institut de statistique de l'Unesco/Montréal/Canada, 2005, 159 p.

Ce recueil de données présente notamment des statistiques mondiales sur les effectifs scolarisés, le personnel enseignant et les mesures de progression et de réussite dans l'enseignement primaire. Des statistiques comparées sur l'espérance de vie scolaire et les dépenses en éducation dans l'enseignement préprimaire, primaire, secondaire et supérieur sont également fournies.

\section{Études de cas}

\section{Afrique}

AMELEWONOU Kokou, BROSSARD Mathieu, GACOUGNOLLE Luc-Charles, La question enseignante dans la perspective de la scolarisation primaire universelle en 2015 dans les pays CEDEAO, CEMAC et PALOP, 2004, 13 pages téléchargées du site http://unesdoc.unesco.org

Les auteurs mettent en évidence le défi que représente l'objectif d'une scolarisation primaire universelle pour les pays du Sud qui devront adapter leur système à une demande qui va exploser, sous la double pression de la démographie en fin de transition et de l'accès généralisé à l'école. 
BARRIER Émilie, L'évaluation, outil de pilotage : étude de cas en Guinée, Revue internationale d'éducation de Sèvres, $n^{\circ}$ 26, juin 2000, pp. 109-119.

Depuis plusieurs années, le ministère de l'éducation guinéen a entrepris une évaluation de l'enseignement primaire pour améliorer la qualité de l'éducation. Il ne s'agit pas simplement de mesurer le niveau des élèves mais également de déterminer les facteurs sur lesquels le ministère peut intervenir pour accroître cette qualité.

BARRIER Emilie, ROBIN Daniel, PASEC. Programme d'analyse des systèmes éducatifs de la CONFEMEN, Évaluation du système éducatif sénégalais: enseignement primaire - annexes, INEADE/Dakar/Sénégal, CIEP/Sèvres/France, 1997, XVIII - 317 p., annexes.

Une évaluation méthodique de l'enseignement primaire au Sénégal a été entreprise par l'INEADE avec le soutien méthodologique du CIEP de 1994 à 1996. L'enquête avait pour objectifs principaux: mesurer le niveau moyen des élèves de $2^{\mathrm{e}}$ et $4^{\mathrm{e}}$ années de l'enseignement primaire et leurs progrès en cours d'année, analyser l'effet du contexte scolaire et familial sur leurs résultats, analyser l'efficacité de l'enseignement et du système éducatif.

BREDA: Bureau régional de l'UNESCO pour l'éducation en Afrique, Scolarisation primaire universelle: un objectif pour tous. Document statistique MINEDAF VIII, UNESCO, Banque mondiale/Washington/États-Unis, Ministère des affaires étrangères/Paris, 2002, 123 p.

La première partie de l'ouvrage dresse un état des lieux des situations de pays africains et des dynamiques observées dans les différents niveaux d'éducation, notamment l'enseignement primaire (taux brut de scolarisation, taux d'accès en $6^{\mathrm{e}}$ ). Après cette présentation du contexte actuel, des recommandations sont faites pour atteindre une scolarisation primaire universelle de qualité en 2015 : mobiliser suffisamment de ressources nationales et faire des arbitrages; optimiser l'utilisation des ressources disponibles pour le primaire; financer les besoins restants. Enfin, des "profils-pays» présentent, pour 53 pays, des indicateurs sur le contexte démographique et macro-économique, des indicateurs de diagnostic, d'autres relatifs à la mobilisation des ressources ou encore aux paramètres structurels de l'éducation primaire.

CONFEMEN: Conférence des ministres de l'éducation des pays ayant le français en partage/Dakar/Sénégal, Les facteurs de l'efficacité dans l'enseignement primaire: les résultats du programme PASEC sur neuf pays d'Afrique et de l'océan Indien, CONFEMEN/Dakar/Sénégal, 1999, 148 p.

En 1991, les ministres de la CONFEMEN (Conférence des ministres de l'éducation nationale) se sont dotés d'un outil d'évaluation, le programme PASEC (Programme d'analyse des systèmes éducatifs). Ses objectifs sont les suivants: identifier des modèles d'écoles efficaces et peu coûteux par la comparaison nationale et internationale; développer, dans chacun des états participants, une capacité interne et permanente d'évaluation; diffuser largement méthodes, instruments et résultats d'évaluation. Ce dernier rapport décrit la situation de l'école primaire dans les neuf pays concernés.

\section{EPT: repères pour l'action. Éducation pour tous en Afrique, UNESCO/Dakar,} 2005, 300 p.

Ce document établit un état des lieux du développement et de l'évolution de chaque cycle d'éducation sur le continent africain et propose une série de projections des conditions actuelles de scolarisation dans le primaire afin de déterminer quels pays seraient susceptibles d'atteindre la scolarisation primaire universelle en 2015. Sont également abordées les options et priorités qui s'offrent aux décideurs africains pour accélérer les progrès pour relever le défi de 2015. 
KO-CHIH TUNG, Assessment of basic education in Sub-Saharan Africa 19902000: Education for All. Report from the Sub-Saharan Region, UNESCO/ Thä̈lande, 2001, 78 pages téléchargées du site http://unesdoc.unesco.org.

Après une présentation de la situation actuelle de l'Afrique subsaharienne en matière d'éducation de base et de son environnement socio-économique, ce rapport rend compte des progrès accomplis depuis 1990 (Conférence mondiale sur l'éducation pour tous, Jomtien) dans ce domaine ainsi que des forces et des faiblesses de l'éducation observées ces dix dernières années. Enfin, il aborde les leçons à tirer de ces observations et fait des recommandations pour les années à venir.

MARCHAND Jacques, Les écoles communautaires - Mali, Sénégal, Togo, IIPE/ Paris, 2000, 209 p. (Programme de recherche et d'études de l'IIPE).

Les États ne parvenant pas à satisfaire la demande de scolarisation, les communautés, rurales pour la plupart, ont créé des écoles avec des moyens réduits, sur le modèle de l'école publique. Il s'agit des écoles d'initiative locale au Togo et des écoles communautaires au Mali. Parfois, l'État incite les communes à prendre en charge des écoles d'un type nouveau où sont tentées des expériences éducatives afin de s'adapter aux besoins de scolarisation. C'est le cas des écoles communautaires de base au Sénégal et des centres d'éducation au développement au Mali. Cet ouvrage offre une vision synthétique des ces différents types d'écoles et s'intéresse à leur développement.

MICHAELOWA Katharina, Teacher job satisfaction, student achievement and the cost of primary education in Francophone sub-Saharan Africa, Hamburg Institute of International Economics/Hamburg, 2002, $n^{\circ} 188,34$ pages téléchargées du site http://opus.zbw-kiel.de

L'auteur propose une analyse empirique de la relation entre satisfaction professionnelle des enseignants, résultat scolaire et coût de l'éducation primaire en Afrique subsaharienne francophone en s'appuyant sur les données recueillies dans le cadre du Programme d'analyse des systèmes éducatifs de la CONFEMEN (PASEC).

MINGAT Alain, Banque mondiale, Deux études pour la scolarisation primaire universelle dans les pays du Sahel en 2015, Banque mondiale/Washington/ÉtatsUnis, 2002, 52 pages téléchargées du site $w w w$-wds.worldbank.org (Série développement humain de la région Afrique).

Dans la perspective de renforcer l'engagement pour l'EPT, les chefs d'État de six pays sahéliens confrontés à des problèmes de nature semblable dans leur système éducatif (Burkina Faso, Guinée, Mali, Niger, Sénégal et Tchad) et des experts en éducation se sont réunis en novembre 2000 à Bamako (Mali). Cette publication comporte les deux documents préparés en amont par la Banque mondiale pour faciliter la discussion. Le premier analyse le développement de l'éducation dans ces six pays durant les quinze dernières années (évolution des scolarisations, financement, efficacité, équité, et principales contraintes pour relever le défis de l'EPT d'ici 2015); le second texte évalue les besoins financiers y compris ceux d'origine externe.

\section{MUKUDI Edith, The Effects of user-fee policy on attendance rates among} Kenyan elementary schoolchildren, International review of education, novembre 2004, vol. 50, $n^{\circ}$ 5/6, p. 447-461.

Cette étude tente de mettre en évidence comment la politique de droit d'inscription au sein de l'éducation primaire au Kenya a affecté les taux de présence et d'interruption de scolarité et comment, de manière plus générale, le gouvernement et le manque de ressources rendent inatteignable l'objectif d'éducation pour tous au Kenya. 
NKAMBA Manasseh, KANYIKA Joe, SACMEQ: Southern African consortium for monitoring educational quality, The quality of primary education: some policy suggestions based on a survey of schools - Zambia, IIPE/Paris, 1998, 81 p., stat., bibliogr. Cette publication présente une étude sur l'enseignement primaire en Zambie et des propositions de politique éducative.

Promoting the transformation of the curriculum for basic education in Mozambique: final report, UNESCO/Paris, 2005, 27 p.

WATT Patrick, Banque mondiale, Community support for basic education in Sub-Saharan Africa, Banque mondiale/Washington/États-Unis, 2001, 78 p., bibliogr. téléchargées du site $w w w$-wds.worldbank.org (Série développement humain de la région afrique)

Après un aperçu historique du rôle de la communauté dans l'éducation africaine, le rapport présente les avantages et les risques qu'implique l'extension du rôle des communautés en éducation et s'interroge sur ce que l'on pourrait attendre des communautés dans ce domaine.

\section{Amérique du Nord}

Conseil supérieur de l'Éducation/Québec, L'organisation du primaire en cycles d'apprentissage: une mise en ouvre à soutenir. Avis au ministre de l'éducation, Conseil supérieur de l'éducation/Sainte-Foy/Canada, 2002, 67 p.

La réforme de l'éducation du Québec, dont le plan d'action a été rendu public en 1996, comprend dans ses dispositifs une organisation de l'enseignement en cycles pluriannuels. Le Conseil supérieur de l'éducation rend public un avis sur les cycles d'apprentissage au primaire.

Ministère de l'Éducation/Québec, Programme de formation de l'école québécoise: version approuvée. Éducation préscolaire. Enseignement primaire, Ministère de l'Éducation/Québec, 2001, 350 pages téléchargées du site www.mels.gouv.qc.ca

Programme de formation de l'éducation préscolaire et primaire québécoise lancé le 24 août 2001.

\section{Amérique latine}

BAESSA Yetilu de, CHESTERFIELD Ray, RAMOS Tanya, Active learning and democratic behavior in Guatemala rural primary schools, Compare: a journal of comparative education, juin 2002, vol. 32, $n^{\circ} 2$, p. 205-218, bibliogr., statistiques À partir d'observations de classes, l'étude s'intéresse aux différences de comportement des enfants selon leur appartenance ethnique et selon leur sexe. Le contexte est celui des établissements guatémaltèques ruraux traditionnels et des établissements où un programme d'apprentissage actif est expérimenté.

UNESCO: Oficina Regional de Educación para America Latina y el Caribe, La conclusión universal de la educación primaria en América Latina : ¿Estamos realmente tan cerca? Informe regional sobre los Objetivos de Desarrollo del Milenio vinculados a la educación, UNESCO/Santiago de Chile, 2004, 58 pages téléchargées $d u$ site http://unesdoc.unesco.org

Ce rapport fait le point sur le chemin parcouru par l'Amérique latine par rapport aux objectifs de développement du millénaire. Des progrès sont notables mais encore insuffisants, 
puisque dans l'ensemble des dix-huit pays de la zone, près de six millions d'adolescents âgés de quinze à dix-neuf ans n'ont pas terminé le cycle d'enseignement primaire. Ils sont plus de $20 \%$ dans ce cas au Guatemala, au Nicaragua et en Honduras. L'étude montre que le difficile accès à l'éducation repose essentiellement sur un problème d'inégalité (sociale, sexuelle, raciale et géographique).

WOLFF Laurence, SCHIEFELBEIN Ernesto, SCHIEFELBEIN Paulina, Primary Education in Latin America: The Unfinished Agenda, Inter-American Development Bank/Washington, 2002, 26 pages téléchargées du site www.iadb.org

L'ouvrage propose de faire le point sur les progrès accomplis depuis une dizaine d'années en matière d'éducation primaire en Amérique latine et plus particulièrement au Brésil, au Chili, en Honduras et au Costa Rica.

\section{Asie}

BEGUM Kamrunnessa, AKHTER Salma, Basic education studies in Bangladesh, UNESCO/Dhaka, 2004, 284 p., illus.

L'ouvrage réunit plusieurs études : l'accueil de la petite enfance, la déscolarisation des adolescents, l'acquisition de compétences équivalentes dans l'éducation formelle et non formelle, le suivi et l'évaluation de l'éducation non formelle, un enseignement primaire de qualité, les perspectives d'emploi des élèves ayant interrompu leur scolarité, le statut des établissements publics et privés et une étude ethnolinguistique de l'arrière-pays de Chittagong.

BRAY Mark, BUNLY Seng, Balancing the books, Basic education in Cambodia, The World Bank/CERC/Hong Kong, 2005, 113 p.

Ce court ouvrage donne des exemples concrets de la reconstruction du système primaire kmer avec l'aide des communautés villageoises.

GOVINDA R., DIWAN Rashm, Community participation and empowerment in primary education, Sage/New Delhi, 2003, 255 p.

Cet ouvrage met en évidence comment la décentralisation de l'enseignement a accru l'importance du rôle joué par la communauté dans le développement de l'éducation primaire en Inde et présente cinq études de cas: Madhya Pradesh, Karnataka, Rajasthan, Bihar and Kerala.

\section{RAMACHANDARAN Vimala, Gender and social equity in primary education:} hierarchies of access, Commission européenne/New Delhi, 2002, 267 p.

L'ouvrage présente des programmes d'éducation primaire et propose six études de cas examinant l'égalité des sexes et l'égalité sociale dans l'accès à l'éducation primaire.

RANGANATHAN Namita, The Primary school child: development and education, Sangam Books/ London, 2000, 226 p.

WILSON John D., UNESCO: Principal Regional Office for Asia and the Pacific/Bangkok, Snapshots of primary and secondary education in AsiaPacific, UNESCO/Thailande, 2001, $n^{\circ} 1,73$ pages téléchargées du site http://unesdoc. unesco.org (Educational innovation for development).

Après un aperçu historique du développement de la coopération en innovation éducative, ce rapport présente différentes initiatives imaginatives qui sont actuellement poursuivies au sein 
de quatorze États membres de l'APEID (Programme d'innovation éducative en vue du développement en Asie et dans le Pacifique): Australie, Bangladesh, Cambodge, Chine, Inde, Laos, Malaisie, Maldives, Myanmar, Népal, Nouvelle-Zélande, Pakistan, Philippines, Thaïlande.

\section{Europe}

DELMOTTE Jean Michel, IVIC Ivan, CHINAPAH Vinayagum, Comprehensive analysis of primary education in the Federal Republic of Yugoslavia, UNICEF/ Belgrade, 2001, 160 p., illus.

Ce document présente les résultats d'une évaluation du sytème formel d'éducation de la République fédérale de Yougoslavie. Ont été plus particulièrement évalués : la structure du système éducatif, les ressources disponibles, l'accès à l'éducation et l'opportunité d'apprendre, la qualité et l'efficacité de l'éducation et enfin le rendement scolaire.

\section{Education for all: The year 2000 assessment Bosnia and Herzegovina national report, UNESCO/Sarajevo, 1999, 23 p., annexes.}

Bilan des réformes menées dans le domaine de l'éducation en Bosnie et Herzégovine et plus particulièrement dans la république de Srpska depuis les années quatre-vingt-dix.

Eurydice: Réseau européen d'information sur l'éducation, Dix années de réformes au niveau de l'enseignement obligatoire dans l'Union européenne (1984-1994), Eurydice/Bruxelles/Belgique, 1997, 315 p.

L'ouvrage présente une analyse comparative des réformes majeures mises en œuvre entre 1984 et 1994 dans les États membres de l'Union européenne et dans les trois pays de l'AELE/EEE. Il décrit ensuite la situation pays par pays concernant la gestion de l'enseignement, la participation sociale, l'évaluation du fonctionnement des établissements, l'organisation de la formation des enseignants, l'intégration des élèves à besoins spéciaux ainsi que les services de soutien et d'encadrement aux écoles.

Eurydice: Réseau européen d'information sur l'éducation, L'enseignement préscolaire et primaire dans l'Union européenne, Eurydice/Bruxelles/Belgique, 1994, 119 p., ill., bibliogr.

Ce document contient des données de base sur l'enseignement des enfants de deux à douze ans dans les systèmes éducatifs des douze pays qui composaient l'Union européenne en 1994. La première partie est consacrée à l'organisation générale: structure de la scolarité, temps scolaire et services d'accueil extra-scolaires. La seconde aborde l'élaboration et le contenu des programmes et l'évaluation des élèves. De nombreux tableaux, graphiques et cartes illustrent le texte.

Eurydice: Réseau européen d'information sur l'éducation, L'enseignement pré-scolaire et primaire dans l'Union européenne, complément à l'étude: la situation en Autriche, en Finlande, en Suède et dans les pays de l'AELE/EEE, Eurydice/ Bruxelles/Belgique, 1994, 63 p.

Complément de l'étude «L'enseignement prescolaire et primaire dans l'Union européenne» (voir ci-dessus), ce document présente des données de base sur l'enseignement préscolaire et primaire en Autriche, en Finlande, en Suède et dans les pays de l'AELE/EEE. 
PRATS Joaquim, RAVENTOS Francesc (Dir.), Los sistemas educativos europeos ¿Crisis o transformación ?, Estudios Sociales, $n^{\circ}$ 18, Obra Social, Barcelone, 2005.

Les problèmes majeurs de l'éducation contemporaine sont examinés à travers les expériences de six pays européens (France, Grande-Bretagne, Allemagne, Pays-Bas, Belgique, Espagne). Une large part est faite à l'école primaire.

YILDIRIM Ali, Instructional planning in a centralized school system : lessons of a study among primary school teachers in Turkey, International review of education, septembre 2003, vol. 49, $n^{\circ} 5$, pp. 525-543.

Cet article présente une étude menée dans deux cent dix écoles sur l'évaluation des pratiques de préparation didactique des enseignants en Turquie et met en évidence les principaux obstacles qu'ils rencontrent: divergence entre curriculum et besoin de la classe, pénurie de temps et de ressources.

\section{L'école primaire en France}

AFAE : Association française des administrateurs de l'éducation, Le système éducatif français et son administration. $10^{e}$ édition, Administration et éducation, 2005, hors série, 327 p.

L'ouvrage qui offre une description du système éducatif français et de son administration, aborde différents aspects de l'enseignement primaire: principes et éléments généraux, organisation administrative des écoles, statut et recrutement des instituteurs et professeur des écoles, dépense d'éducation.

BOTTIN Yves, Enseigner en école, un métier pour demain : rapport au ministre de l'Éducation nationale, Ministère de l'Éducation nationale, Paris, 2002, 54 pages téléchargées du site $w w w$.ladocumentationfrancaise.fr

Après un état des lieux présentant le métier d'enseignant du premier degré, les évolutions éducatives et sociales, les attentes de la société et de l'institution scolaire française, le rapport présente plusieurs propositions relatives à la réorganisation de l'école et son réseau, à l'amélioration des conditions de travail, à la prise en compte de nouveaux besoins dans la formation des maîtres et à l'évaluation des enseignants.

CROS Françoise, OBIN Jean-Pierre, Attirer, former et retenir des enseignants de qualité: rapport de base national de la France, Documentation française/Paris, 2004, 106 pages et bibliographie téléchargées du site www.oecd.org

Ce rapport étudie la situation de la France quant au recrutement d'enseignants et met en évidence la structure d'âge de la population française peu favorable à la satisfaction des besoins importants de recrutement, dus aux départs nombreux à la retraite dans les années à venir.

DASTE Pierre dir., Administrer l'école primaire, Hachette Education/Paris, 1998, 478 p., bibliogr., lexique (Former organiser pour enseigner)

L'ouvrage expose et commente l'ensemble des règles d'organisation de l'enseignement du premier degré. Il permet de faire le point sur le fonctionnement de l'école (rôle du directeur, relations avec les autorités de tutelle et les communes, enseignement spécialisé), la gestion de ses personnels et la composition des programmes. 
DELEVOYE Jean-Paul, LABADIE Francine, Pour une approche globale du temps de l'enfant: rapport d'évaluation du Comité d'évaluation et de suivi des aménagements des rythmes de l'enfant, La Documentation française, Paris, 1999, $376 p$.

Synthèse des résultats des études et productions nationales ainsi que des principales évaluations locales en matière d'aménagement du rythme de l'enfant.

DUHAMEL Marcel, HOUCHOT Alain, RICHON Henri-Georgeset al., L'évolution du réseau des écoles primaires: rapport définitif, IGEN, Paris, 2003. 71 pages téléchargées $d u$ site $w w w$.ladocumentationfrancaise.fr

Faisant suite à une enquête sur l'organisation, la structure des écoles, le fonctionnement effectif des différents réseaux et la politique départementale envisageable, ce rapport fait l'examen critique des textes et des politiques suivies.

FEROLE Jean, Guide de législation et de réglementation scolaire : école primaire, Hachette Education/Paris, 2004, 366 p. (Guide pratique)

Organisé en fiches brèves, cet ouvrage présente la réglementation scolaire de l'école primaire sous forme de questions et de réponses et fait le point sur l'organisation actuelle de l'école du premier degré maternelle et élémentaire.

FERRIER Jean, Améliorer l'efficacité de l'école primaire: rapport remis à $M^{m e}$ la ministre déléguée chargée de l'enseignement scolaire, Ministère de l'Éducation nationale, Paris, 1998, 148 pages téléchargées du site www.ladocumentationfrancaise.fr

Après un état des lieux présentant les résultats et les moyens de l'école, le rapport fait des propositions quant à l'organisation pédagogique.

FERRIER Jean, Les inspecteurs des écoles primaires 1835-1995: ils ont construit l'école publique, L'Harmattan/Paris, 1997, 965 p. en 2 vol., bibliogr., (Education et formation. Références).

L'ouvrage retrace l'histoire des inspecteurs de l'Éducation nationale depuis leur combat pour imposer leur autorité face à l'Église, aux notables, aux délégués cantonaux (1835-1880); le rôle essentiel qu'ils ont joué dans la construction de l'école de la République de 1880 à 1940 et leurs incertitudes actuelles en ce qui concerne leurs missions.

LAHIRE Bernard, Culture écrite et inégalités scolaires : sociologie de l'échec scolaire à l'école primaire, Presses universitaires de Lyon/Lyon/France, 2000, 310 p., bibliogr.

Estimant le sujet peu abordé jusqu'à présent, l'auteur souhaite contribuer à la compréhension de «l'échec scolaire» à l'école primaire. Méthodologiquement, il recourt à une démarche du type socio-historique basant notamment la réflexion sur les différences entre cultures écrites et cultures orales. Il s'appuie sur une observation des pratiques et productions scolaires en accordant une place privilégiée au problème des cultures écrites.

\section{LELIÈVRE Claude, L'école obligatoire: Pour quoi faire? Une question trop} souvent éludée, Retz, 2004, 142 p. (Défis d'éducation).

L'auteur retrace les grandes étapes qui ont marqué l'évolution du système éducatif jusqu'à la naissance du collège unique à travers une réflexion sur les objectifs fondamentaux de l'école telle qu'elle existe depuis la fin du XIX ${ }^{\mathrm{e}}$ siècle. 


\section{LURÇAT Liliane, La destruction de l'enseignement élémentaire et ses penseurs,} De Guibert, 1998, 241 p. (Guide pratique).

L'auteur considère que les échecs scolaires de plus en plus nombreux sont dus au nouveau système des apprentissages fondamentaux : lecture, écriture, calcul. En s'appuyant sur de nombreux exemples, elle cherche à décrire les mécanismes idéologiques à travers lesquels un certain nombre de penseurs, à la fin des années soixante, ont entrepris la destruction systématique des grandes bases de l'enseignement élémentaire.

MASCLET Georges dir., GUILLEMARD Jean-Claude, SIX Francis et al., Écoles et management, Aubin/Saint-Etienne, 2001, 203 p.

Cet ouvrage qui réunit plusieurs contributions, a pour objectif de fournir aux responsables des écoles et aux psychologues scolaires un outil pour comprendre le management et l'organisation du système éducatif français, particulièrement de l'école élémentaire. Après une analyse systémique de l'organisation des écoles primaires, il présente l'analyse institutionnelle et la psychologie des organisations.

Ministère de l'Education nationale. Conseil national des programmes, $\mathbf{Q}$ 'apprendon à l'école élémentaire? 2003-2004. Les programmes, SCEREN-XO éditions/ Paris, 2003, $349 p$.

Les nouveaux programmes pour l'école primaire, élaborés au terme d'une large concertation et mis en place à partir de la rentrée 2002, sont le fruit d'un groupe d'experts présidés par le recteur Philippe Joutard avec la contribution du Conseil national des programmes. Cette publication présente les programmes et, en complément, des documents d'application et d'accompagnement apportant des indications concrètes sur la manière dont ces programmes peuvent être mis en œuvre.

PERETTI Claudine dir., Ministère de la Jeunesse de l'Education nationale et de la recherche. DEP: Direction de l'évaluation et de la prospective/Paris, Repères et références statistiques sur les enseignements, la formation et la recherche : 2005, Ministère de l'Education Nationale. DEP/Direction de l'évaluation et de la prospective, 2005, 384 p. (RERS).

L'ouvrage présente notamment des données statistiques sur les établissements, les élèves, les enseignants, et le financement du premier degré. 
